men deshalb so schwierig sei, weil in Tarifverhandlungen stets nur sehr begrenzter Verteilungsspielraum bestehe. Die nach einer diskriminierungsfreien Arbeitsbewertung zu erwartenden höheren Kosten für die Arbeitgeber müssten durch einen weitgehenden Verzicht auf eine generelle Tariferhöhung kompensiert werden. Hierfür gebe es in den Tarifkommissionen oft keine Mehrheit.

Anja Weusthoff vom Bundesvorstand der Gewerkschaft der Polizei wies darauf hin, dass Frauen bei der Polizei in Beförderungsrunden oft das Nachsehen haben und nicht in höhere Besoldungsgruppen aufsteigen, weil sie, obwohl sie mindestens so gut qualifiziert sind wie ihre männlichen Kollegen, generell schlechtere Dienstpostenbewertungen erhalten. $\mathrm{MdB}$ Andrea Voßhoff versprach spontan, diesbezüglich eine Anfrage an das Innenministerium zu richten.

Zur Frage aus dem Publikum, warum Personalräte in $\mathbb{S} 17$ Abs. $2 \mathrm{AGG}^{1}$ keine Erwähnung finden, versprach MdB Cornelia Möhring, eine kleine Anfrage an die Bundesregierung zu richten. Diese ist inzwischen gestellt und beantwortet worden. ${ }^{2}$

In den Diskussionsbeiträgen wurde deutlich, dass die Antworten der Politikerinnen viele der anwesenden Juristinnen nicht zufriedengestellt haben. Aus dem Publikum wurde wiederholt und teilweise mit deutlicher Verärgerung darauf hingewiesen, dass Abwarten keine Option sei und dass der Gesetzgeber endlich handeln müsse, um den rechtswidrigen $\mathrm{Zu}$ stand der Entgeltungleichheit zwischen Frauen und Männern auch in Deutschland zu beseitigen.
Immerhin haben einige der dem Bundestag angehörenden Politikerinnen versprochen, mit dem djb in Kontakt zu bleiben, um gemeinsam parlamentarische Initiativen mit dem Ziel vorzubereiten, das Gebot des gleichen Entgelts für Frauen und Männer bei gleicher und gleichwertiger Arbeit effektiv umzusetzen.

Zum Schluss der interessanten Veranstaltung, die gezeigt hat, dass zum Thema Entgeltgleichheit strukturelle Lösungen unabdingbar und dringend notwendig sind, dankte djb-Präsidentin Jutta Wagner allen Teilnehmerinnen auf dem Podium sowie Prof. Dr. Heide Pfarr für ihre sehr gelungene Moderation. Ihr Dank galt insbesondere auch der Thüringer Gleichstellungsbeauftragten Johanna Arenhövel, die neben einleitenden Worten Grüße der Thüringer Landesregierung, insbesondere der ersten Thüringer Ministerpräsidentin Christine Lieberknecht MdL überbracht hatte.

\footnotetext{
1 In Betrieben, in denen die Voraussetzungen des $\S 1$ Abs. 1 S. 1 des Betriebsverfassungsgesetzes vorliegen (Betriebe mit in der Regel mindestens fünf ständigen bei einer Betriebsratswahl wahlberechtigten Arbeitnehmer(innen), von denen drei wählbar sind), können bei einem groben Verstoß des Arbeitgebers gegen Vorschriften aus diesem Abschnitt (gemeint ist Abschnitt 2 des AGG, aus dem sich auch das Verbot der Entgeltdiskriminierung ergibt) der Betriebsrat oder eine im Betrieb vertretene Gewerkschaft unter der Voraussetzung des $\S 23$ Abs. 3 S. 1 BetrVG die dort genannten Rechte geltend machen. ( $\$ 17$ Absatz 3 AGG stellt klar: Mit dem Antrag dürfen nicht Ansprüche der Benachteiligten geltend gemacht werden).

2 BT-Drucksache 17/4003, <http://dip21.bundestag.de/dip21/ btd/17/040/1704003.pdf> (Zugriff 3.1.2011).
}

\title{
Begrüßung zur djb-Veranstaltung zum Thema Entgeltungleichheit
}

\section{Juristinnen fragen - Politikerinnen antworten, Podiumsdiskussion am 25. November 2010 in der Vertretung des Freistaats Thüringen beim Bund, Berlin}

\section{Jutta Wagner \\ Präsidentin des djb; Rechtsanwältin und Notarin, Berlin}

Es freut mich keineswegs, Sie hier heute im Namen des djb zu einer Veranstaltung über Entgelt(un)gleichheit begrüßen zu dürfen. Was sollte daran erfreulich sein, im Jahr 2010, 61 Jahre nach Inkrafttreten des Grundgesetzes mit dem Gleichheitsgebot des Artikels 3 und 62 Jahre nach der Gründung des djb immer noch und immer wieder darüber diskutieren zu müssen, welches der richtige und schnellste Weg zur Beseitigung des Gender Pay Gap in Deutschland sein könnte? Oder täuschen wir uns und es besteht, wie uns das Institut der Deutschen Wirtschaft am 9. November 2010 mitteilte, gar kein Anlass mehr zur Aufregung? Einer Berliner Tageszeitung immerhin war diese Nachricht den prominentesten Platz auf der ersten Seite wert. Verhält Frau sich richtig und ersetzt einfach ihre weibliche Berufsbiografie durch eine männliche, kann sie es immerhin theoretisch schaffen, den Lohnabstand von 29 Prozent auf vier Prozent zu minimieren. Das ist doch einmal eine gute Nachricht. Nur hat sie leider mit der Realität im Arbeitsleben wenig zu tun:

- Die sieht nämlich nach wie vor so aus, dass die berufliche Qualifikation von Mädchen überwiegend für nicht so wichtig gehalten wird.

- Die sieht nach wie vor so aus, dass Frauen die Kinder bekommen - wenn sie es denn wagen -, ihre Erwerbstätigkeit betreuungs- oder pflegebedingt unterbrechen oder aufgeben, in Teilzeit oder in den so niedlich „Minijobs“ genannten Armutsfallen landen.

Dies ist aber nur ein Teil des Problems. Der andere, mindestens ebenso wichtige Teil besteht darin, dass Entgeltdiskriminierung bei gleicher oder gleichwertiger Arbeit für einen nicht 
unerheblichen Teil des Gender Pay Gaps veranwortlich ist. Daran haben die bisher eingesetzten Instrumente nichts ändern können.

Insofern freut es mich dann doch, Sie heute hier zu unserer Veranstaltung begrüßen zu dürfen. Es freut mich deshalb, weil wir wohl kaum mehr juristischen Sachverstand zu diesem Thema finden können, als er heute hier präsent ist und weil wir in der Sache selbst engagierte Politikerinnen aller Bundestagsfraktionen hier haben, mit denen wir hoffentlich an einem Strang und den festgefahrenen Karren endlich aus dem Dreck ziehen können.

Zunächst einmal aber darf ich Sie, Frau Arenhövel, ganz besonders herzlich begrüßen. Als Thüringerin sind Sie, Frau Arenhövel, in Ihrer Landesvertretung doch zu Hause und so darf ich Ihnen zunächst dafür danken, dass wir unsere Veranstaltung in Ihrer Landesvertretung durchführen dürfen. Sie waren seit 1990 bis 2004 Mitglied des Thüringer Landtags und unter anderem Sprecherin für Soziales, Familie und Gesundheit der CDU-Landtagsfraktion. Im Thüringer Ministerium für Soziales, Familie und Gesundheit sind Sie seit dem 1. August 2004 Beauftragte für die Gleichstellung von Frau und Mann. Mit dem djb in Thüringen haben Sie u.a. zu Frau- enhäusern und zum Thüringer Gleichstellungsgesetz zusammengearbeitet und sind dort ein gern gesehener Vortragsgast. Ich freue mich ganz besonders, dass Sie sich gerade heute die Zeit genommen haben, zu uns zu kommen, denn in Thüringen findet heute der Aktionstag „Nein zu Gewalt gegen Frauen“ statt und ich bin sicher, dass Sie in Thüringen heute bei mehr als einer Veranstaltung sehr willkommen gewesen wären. Wir freuen uns jetzt alle auf Ihr Grußwort.

Prof. Dr. Marlene Schmidt ist Rechtsanwältin und zugleich Professorin für Arbeitsrecht an der Universität Frankfurt am Main. Seit ungefähr 20 Jahren arbeitet sie ausschließlich im Arbeitsrecht, im individuellen und im kollektiven. Sie berät und vertritt ausschließlich Arbeitnehmer(innen), Betriebs- und Personalräte, Gewerkschaften und Arbeitnehmerkoalitionen. Unter ihren Veröffentlichungen finden sich auch Beiträge zum Grundsatz des gleichen Entgelts für Frauen und Männer. Nicht zuletzt ist Frau Prof. Schmidt seit vielen Jahren Mitglied im djb und unserer Kommission Arbeits-, Gleichstellungs- und Wirtschaftsrecht, liebevoll auch „K1 “ genannt. Sie wurde von der Mitgliederversammlung des djb 2009 zu deren Vorsitzenden gewählt. Ich darf Sie, Dich, liebe Marlene Schmidt, jetzt um Dein Statement bitten.

\section{Statement zum Thema Entgeltungleichheit}

\section{Juristinnen fragen - Politikerinnen antworten, Podiumsdiskussion am 25. November 2010 in der Vertretung des Freistaats Thüringen beim Bund, Berlin}

\section{Prof. Dr. Marlene Schmidt}

Vorsitzende der Kommission Arbeits-, Gleichstellungs- und Wirtschaftsrecht des djb; Rechtsanwältin, Frankfurt am Main

Meine sehr verehrten Damen, liebe Kolleginnen,

der Deutsche Juristinnenbund bedankt sich, dass Sie sich die Zeit genommen haben, um heute hier mit uns zu diskutieren. Dank auch der Thüringischen Landesvertretung, dass wir uns heute hier in diesen schönen Räumen treffen können.

Das Thema, mit dem wir uns befassen müssen, ist allerdings ein großes Ärgernis. Denn das Grundgesetz bestimmt seit über 60 Jahren: Männer und Frauen sind gleichberechtigt. Und der EG-Vertrag besagt seit mehr als 50 Jahren: Jeder Mitgliedstaat stellt die Anwendung des Grundsatzes des gleichen Entgelts für Männer und Frauen bei gleicher oder gleichwertiger Arbeit sicher. Hierbei handelt es sich auch nicht lediglich um Verfassungslyrik oder Programmsätze, sondern um unmittelbar geltende Grundrechte, die auch im Verhältnis zwischen Privaten Geltung entfalten. Die Durchsetzung und die Verwirklichung von Entgeltgleichheit gehören daher zu den Themen, die auf der Prioritätenliste des Deutschen Juristinnenbundes ganz oben stehen.

\section{Der sogenannte „Gender Pay Gap“ und seine Ursachen}

Der Gender Pay Gap, das heißt der prozentuale Unterschied im durchschnittlichen Bruttostundenverdienst von Frauen und Männern, lag in Deutschland mit 23,2 Prozent auch im Jahr 2008 deutlich über dem Durchschnitt der Europäischen Union (18\%). Wie das Statistische Bundesamt (Destatis) anlässlich des Internationalen Frauentages am 8. März 2010 mitteilte, wiesen von den 27 Ländern der europäischen Union lediglich Estland (letzter Wert für 2007: 30,3\%), die Tschechische Republik $(26,2 \%)$, Österreich $(25,5 \%)$ und die Niederlande (letzter Wert 2007: 23,6\%) einen gegenüber Deutschland höheren geschlechtsspezifischen Verdienstabstand auf.

Dieser Verdienstabstand ist - so das Institut für Arbeitsmarkt- und Berufsforschung - teilweise auf unterschiedliche Merkmale von Frauen und Männern wie etwa das Ausbildungsniveau zurückführen, aber auch auf ungleiche Erwerbsverläufe und Charakteristika von Betrieben mit hohem Frauen- bzw. Männeranteil. Durch solche Faktoren lassen sich die festgestellten Verdienstunterschiede allerdings nicht in vollem Umfang erklären, es verbleibt ein nicht unerheblicher Rest.

So hat in Deutschland auch im Zeitraum 1993 bis 2001 ein relativ konstanter Verdienstunterschied von 12 Prozent auch zwischen solchen Frauen und Männern bestanden, die sich weder in der Ausbildung noch in Alter und Beruf unterschei- 